KURZ GEMELDET

Allergy News

\section{Cave: Lammungsohren!}

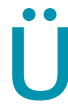
ber eine außergewöhnliche Berufskrankheit bei englischen Schafzüchtern berichten Ärzte aus Southampton: Die betroffenen Farmer entwickeln ausschließlich zur Lammungszeit im Frühjahr an jeder Ohrmuschel etwa vier bis sechs Blasen, die anschließend verkrusten. Sind nach zwei bis drei Monaten alle Lämmer geboren, gehen die Hauteruptionen wieder zurück. Außer den Ohrmuscheln ist kein weiteres Hautareal betroffen, histologisch finden sich perivaskuläre T-Zelldominierte lymphozytäre Infiltrate. Die Inzidenz der Hauterscheinungen korreliert mit Häufigkeit und Intensität des Schaf-

\title{
Angioödemtherapie in Sicht
}

$\mathrm{P}$ atienten mit hereditärem Angioödem (HAE) weisen meist eine Mutation eines Einzelallels für den C1-Inhibitor (C1-INH) auf. C1-INH ist ein Suppressor der Kallikrein-Kinin-Kaskade. Es ist bekannt, dass die HAE-Symptome auf einer Überaktivierung der Kallikrein-Kinin-Kaskade beruhen, wodurch vermehrt Bradykinin freigesetzt und damit das Ödem induziert wird. Es wird vermutet, dass bei HAE-Patienten Stress oder Traumata die endogenen C1-INH-Spiegel unter einen kritischen Punkt absinken lassen, wodurch die Kallikrein-Kinin-Kaskade nicht mehr ausreichend inhibiert ist.

\section{Hypoallergene Hühnereier}

auptallergene im Hühnerei sind

Ovomucoid, Ovalbumin, Ovotransferrin und Lysozym, die fast $80 \%$ der Eiklar-Trockensubstanz ausmachen. Mit den üblichen Verfahren zur Nahrungsmittelbearbeitung konnte deren Allergenität bisher nicht reduziert werden, ohne den Geschmack und die Textur des AusFoto: miya

Enzym-Verfahren entwickelt, bei dem die Allergenität des pasteurisierten Eiklars sukzessive auf rund ein 1/100 des Ausgangsmaterials reduziert wurde. Verarbeitet in Gerichten wie Reiskuchen oder Pudding war die neue hypoallergene Eizubereitung in Geschmack oder Textur nicht gangsmaterials gänzlich zu verändern. Im Rahmen des von der Europäischen Union unterstützen Projekts REDALL (Reduced Allergenicity of Processed Food) wurde jetzt ein neunstufiges, kombiniertes Hitze-

Hildebrandt $S$ et al. J Agric Food Chem 2008; 56: 1727-33

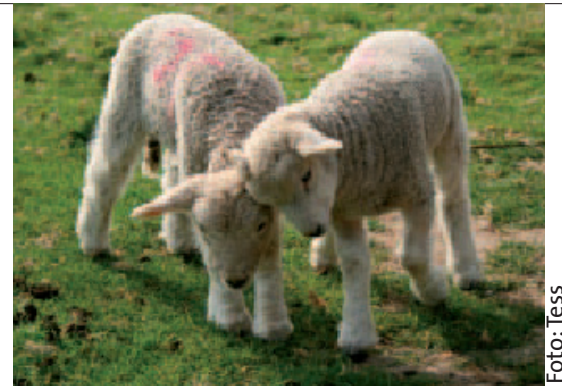

kontakts der Farmer. Möglicherweise handelt es sich um eine spezielle Form der Frühlingsdermatitis, die durch schafspezifische Substanzen getriggert wird. Trotz mehrerer Nachfragen in einschlägigen Fachblättern scheinen Schafzüchter auf der Südhalbkugel diese „Lammungsohren“ allerdings nicht zu kennen.

$b k$

Heathcote $\mathrm{K}$ et al. Br J Dermatol 2008; 158: 134-7
In einer randomisierten Studie wurde diese Annahme nun klinisch bestätigt und gleichzeitig Hoffnung auf ein wirksames Therapeutikum geweckt: Ecallantid ist ein rekombinantes Protein, das spezifisch Kallikrein inhibiert, damit auch Bradykinin blockiert und so die akute Symptomatik von HAE-Patienten beeinflusst. Intravenös gegeben konnte der Kallikrein-Inhibitor signifikant besser als Plazebo die Symptome einer HAE-Attacke innerhalb von vier Stunden lindern.

$b k$

Schneider L et al. J Allergy Clin Immunol

2007; 120: 416-22

\section{Zöliakie im Kuhstall}

7 öliakie-Patienten müssen sich glutenfrei ernähren. Je nach Empfindlichkeit der Patienten auf die Getreideeiweiße kann eine solche Diät ausreichend sein, bei manchen muss die "Glutendiät" aber auch auf die Atemluft ausgedehnt werden. Dies war der Fall bei zwei Farmarbeitern, die trotz einer glutenfreien Diät symptomatisch waren. Der Grund: Die Männer verfütterten regelmäßig eine Gerste-MaisWeizen-Kraftmischung an junge Kälber und inhalierten bzw. verschluckten beim Anrühren des Breis rechnerisch etwa $150 \mathrm{~g}$ glutenhaltige Staubpartikel pro Tag. Erst das Tragen einer Gesichtsmaske während der Stallarbeit reduzierte die Symptomatik.

Kasim S et al. N Engl J Med 2007; 356: 2548-9

\section{Asthma durch Reiniger?}

W as aus dem professionellen Reinigungsgewerbe bereits bekannt ist, wurde nun auch im privaten Bereich beobachtet: Die Benutzung von Reinigungsmitteln löst Asthma aus, so die Ergebnisse einer Neun-Jahres-Followup-Studie. Dies galt allerdings nur für Reinigungssprays, für die sogar eine Dosis-Wirkungs-Beziehung hergestellt wurde: Bereits der einmal wöchentliche Einsatz der bequemen Haushaltshelfer erhöhte das Asthmarisiko leicht, sehr hohe Raten an neu diagnostiziertem Asthma ergaben sich bei mindestens viermal wöchentlicher Anwendung - unabhängig vom Atopiestatus der Patienten. Die Sprays zur Reinigung von Fenstern, Möbeln und Kacheln enthalten u. a. Alkohole, Ammoniak, Chlorid freisetzende Substanzen, Glykolether und Acrylpolymere, die als Aerosol eingeatmet wahrscheinlich zu einer Entzündungsreaktion der Bronchien führen.

red
Zock JP et al. Am J Respir Crit Care Med 2007; 176: 735-41 